\title{
Graphene to graphite: electronic changes within DFT calculations
}

\author{
A.Z. AlZahrani and G.P. Srivastava \\ School of Physics, University of Exeter, Stocker Road, Exeter EX4 4QL, U.K.
}

(Received on 22 August, 2009)

\begin{abstract}
Calculations based on the first-principles pseudopotential plane-wave method and density-functional theory are performed to investigate the electronic properties of graphene, bilayer graphene, multilayer graphene, and graphite. From an analysis of the electronic band structure close to the Fermi level, we have quantified the gradual change in the Fermi surface topology from the point-like structure for graphene to a warped triangular shape for graphite. We have also discussed the gradual change in the electron and hole effective masses and velocities as the system evolves from graphene to graphite.
\end{abstract}

Keywords: Graphene; Bilayer graphene; Multilayer graphene; Graphite; Density functional theory; Local density approximation; Pseudopotential theory; Fermi surface; Electronic structure

\section{INTRODUCTION}

Recently, carbon-based materials such as graphene (a single hexagonal layer of carbon atoms) and graphite (threedimensional hexagonal structure of carbon atoms) have generated a lot of interest due to their exotic electronic properties $[1,2]$. From the electronic structure point of view, monolayer graphene has a linear band dispersion near the corner (K) point of its Brillouin zone originating from the $\pi$ and $\pi^{*}$ bondings between the two carbon basis atoms within the hexagonal unit cell. This results in carriers (electrons and holes) to behave like massless Dirac particles with very large Fermi velocity [3]. The carrier mass and velocity are found to change strongly with the increase in the number of atomic layers in a graphene sample [3]. The basic understanding of the electronic band structure and the Fermi surface topology of graphite has been gained for a long time [4-8]. McClure [5] has described the energy band structure of graphite close to the Fermi level using the Slonczewski-Weiss model. This work has revealed that the electron and hole Fermi surfaces are highly elongated and aligned along the highsymmetry edges of the Brillouin zone. The band structure calculations obtained by Zunger [7] have shown that the electrons are confined to narrow holes close to the Brillouin zone edges. Moreover, Tatar and Rabii [8] have reported that the constant-energy Fermi surfaces of graphite show clear symmetry of the bands above and below the Fermi level.

Despite these basic understandings, an important area that has not been investigated thoroughly is the change in the key electronic parameters, such as carrier effective mass, carrier velocity, and Fermi surface topology, as the system evolves from the strictly two-dimensional $\left(\mathrm{sp}^{2}\right)$ graphene structure to the three-dimensional $\left(\mathrm{sp}^{2}+\mathrm{p}_{z}\right)$ graphite structure. For a full understanding of the origin of these changes, it is of particular importance to thoroughly investigate the basic electronic structure such as the dispersion of the band structure near the high-symmetry edges (such as $\mathrm{K}$ and $\mathrm{H}$ points) and the Fermi surface at these particular points.

In this paper, we present a first-principles theoretical investigation of the electronic band structure of graphene, bilayer graphene, multilayer graphene, and graphite. From these calculations, the effective masses and velocities of the carriers have been estimated, and compared with available experimental measurements, for each of these structures. An attempt has been made to explain the gradual change in the electronic properties from the two-dimensional graphene structure to the three-dimensional graphite structure.

\section{COMPUTATIONAL METHOD}

We have performed first-principles calculations within the density functional theory [9] using the local density approximation (DFT-LDA). The Perdew-Zunger exchangecorrelation scheme [10] was considered to treat the electronelectron interaction. The electron-ion interaction was treated by using the ultrasoft pseudopotential for carbon [11]. The single-particle Kohn-Sham [12] wave functions were expanded in the framework of a plane wave basis set with a kinetic energy cutoff of 60 Ryd. Self-consistent solutions of the Kohn-Sham equations were obtained by employing the $14 \times 14 \times 14$ and $14 \times 14 \times 4$ k-points Monkhorst-Pack sets [13] within the Brillouin zones for graphite and other structures, respectively. For Brillouin zone integration the eigenvalues at the special $\mathbf{k}$ points were thermally smeared by using the Methfessel-Paxton scheme [14] with a broadening factor of 0.00002 Ryd. These parameters have been carefully chosen after several convergence test calculations.

We adopted a supercell technique for modelling the multilayer graphene. A supercell contained seven atomic layers of carbon atoms and a vacuum region equivalent to eight atomic layers. Relaxed atomic positions were obtained by using the total-energy and force minimisation methods. The equilibrium atomic positions were determined by relaxing atoms in all except the eighth layer, which was kept in its bulk position.

\section{RESULTS AND DISCUSSION}

\section{III.1. Bulk graphite}

We made a series of total energy calculations with different choices of the in-plane lattice constant $a$ and the ratio $c / a$ of the out-of-the plane to the in-plane lattice constants. From our calculations we determined that the minimum energy configuration results for the choices $a=2.448 \AA$ and $c / a=2.711$. These values are in agreement with several previous ab initio calculations $[15,16]$.

The band structure for graphite close to the Fermi level, 
and along the inter-planar direction (the KH direction in the Brillouin zone), is shown Fig. 1(a). Within about $1 \mathrm{eV}$ around the Fermi level we have four electronic bands, two derived from $\pi$ orbitals and two from the $\pi^{*}$ orbitals of the basis atoms. The inner pair of the $\pi / \pi^{*}$ bands is degenerate and shows very little dispersion along the $\mathrm{KH}$ direction in the Brillouin zone. This pair of bands is electron-like and holelike towards the $\mathrm{K}$ and $\mathrm{H}$ points, respectively. The width of the electron-like part of the band is very slightly wider than the width of the hole-like part. Also, since the band crosses the Fermi level at approximately $\mathrm{KH} / 3$, it indicates that the hole state is more elongated than the electron state. These results are in agreement with previous first-principles works (see, e.g. Ref. [8]). The outer pair of the $\pi / \pi^{*}$ bands are dispersive along the KH direction, with the $\pi$ and $\pi^{*}$ bands dispersing up and down the energy axis, respectively. Our calculated energy gap between the $\pi$ and $\pi^{*}$ bands is $0.07 \mathrm{eV}$ at the $\mathrm{H}$ point and $1.41 \mathrm{eV}$ at the $\mathrm{K}$ point.
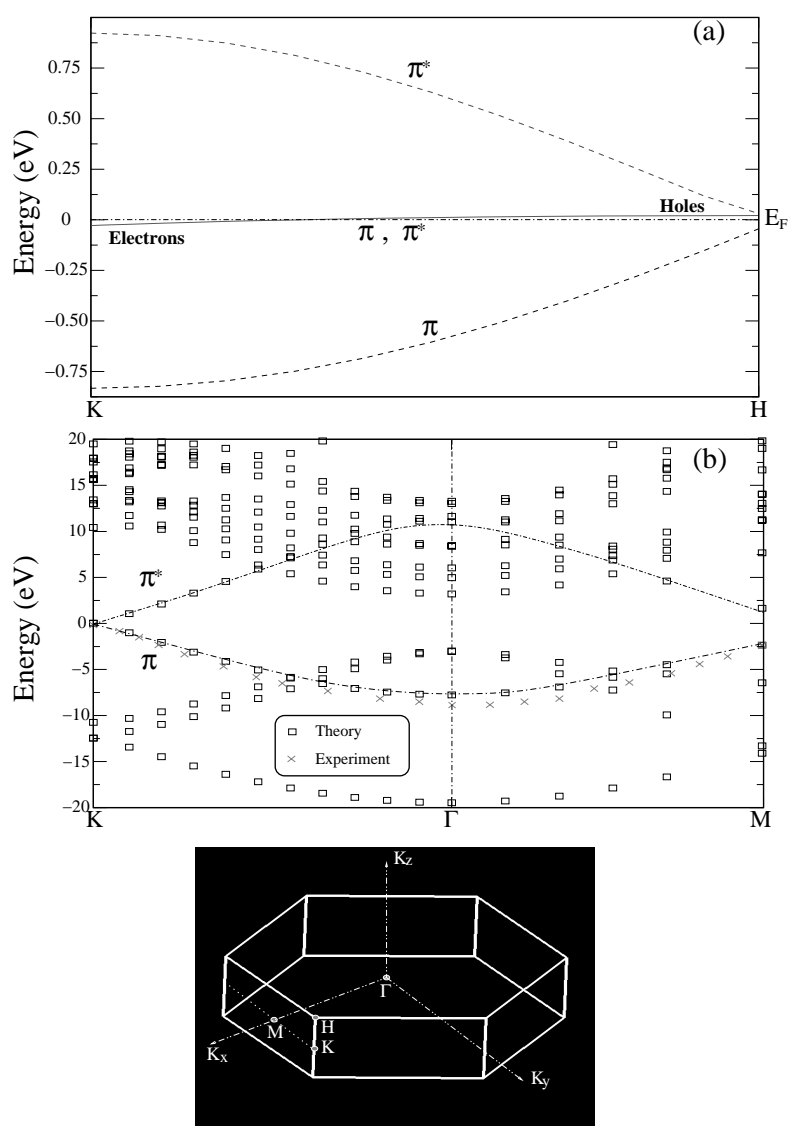

FIG. 1: The band structure plot of (a) bulk graphite along the KH direction and close to the Fermi level and (b) monolayer graphene along the high-symmetry directions (KГM). The hexagonal Brillouin zone is also shown.

In Fig. 2(a) we have plotted the in-plane dispersion of the electronic bands near the Fermi level. [We performed calculations along several in-plane directions, and found similar results.] An attempt to fit such curves close to the
$\mathrm{K}$ point suggests a purely quadratic behaviour, indicating that the carriers are normal (massive) electrons. From these calculations we estimated the effective electron mass value $m^{*}=0.043 m_{e}$. This value is consistent with the reported theoretical value of $0.045 m_{e}[8,17]$ and close enough to the experimental result of $0.06 m_{e}$ [18]. We also calculated the electron (in-plane) velocity of the magnitude $0.94 \times 10^{6} \mathrm{~m} / \mathrm{s}$. This result is in good agreement with the angle-resolved photoemission data obtained by Zhou et al. [19] who obtained a value of $0.91 \times 10^{6} \mathrm{~m} / \mathrm{s}$. When fitting the in-plane dispersion of hole bands we needed to include both the linear and quadratic terms. This suggests that the behaviour of holes can be described as a mixture of Dirac-like (massless) and normal (massive) particle. Our conclusions regarding the quasi-particle nature of the electrons (as normal) and holes (as a mixture of normal and Dirac-like) near the Fermi level in graphite support the phase analysis of quantum oscillations [20] and the quantum Hall effect [21].
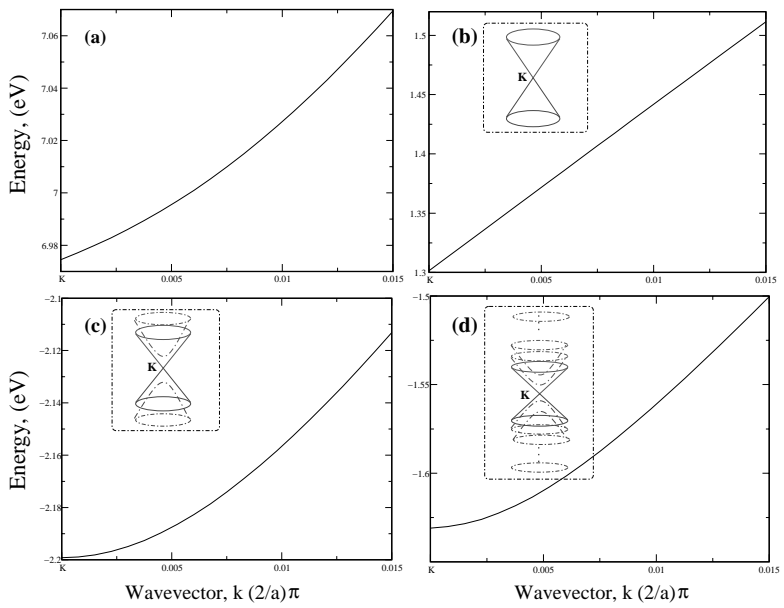

FIG. 2: The in-plane electronic band structure for the innermost $\pi^{*}$ band near the the $\mathrm{K}$ point for (a) graphite, (b) monolayer graphene, (c) bilayer graphene, and (d) multilayer (7-layer) graphene. The insets indicate the dispersion curves for the outer pairs of the $\pi / \pi^{*}$ bands.

The shape and symmetry of the Fermi surface in graphite has long been a topic of discussion, with contradictory views in the 1950s [22], 1960s [6] and 1970s [23]. First-principles band structure calculations by Tatar and Rabii in 1982 [8] provide the accepted picture for the band structure, and shape and symmetry of the Fermi surface. It should be noted that Tatar and Rabii constructed the Fermi surface by adopting two parametrized fitting procedures of bands. In our work the Fermi surface was mapped without the need for any parameterization of bands. Our calculations have presented results, shown in panel (a) of Figs. 3 and 4, in close agreement with those by Tatar and Rabii. We find that the Fermi surface is elongated along the KH direction. The shape of the Fermi surface can be described as a warped and squeezed cylinder, with almost equal width at the $\mathrm{K}$ and $\mathrm{H}$ points. The squeezed feature can be seen as a 'neck' at KH/3. The warped triangular cross-sections at the $k_{z}=0$ and $k_{z}=\mathrm{KH}$ heights can be clearly noticed in Fig. 4(a). 

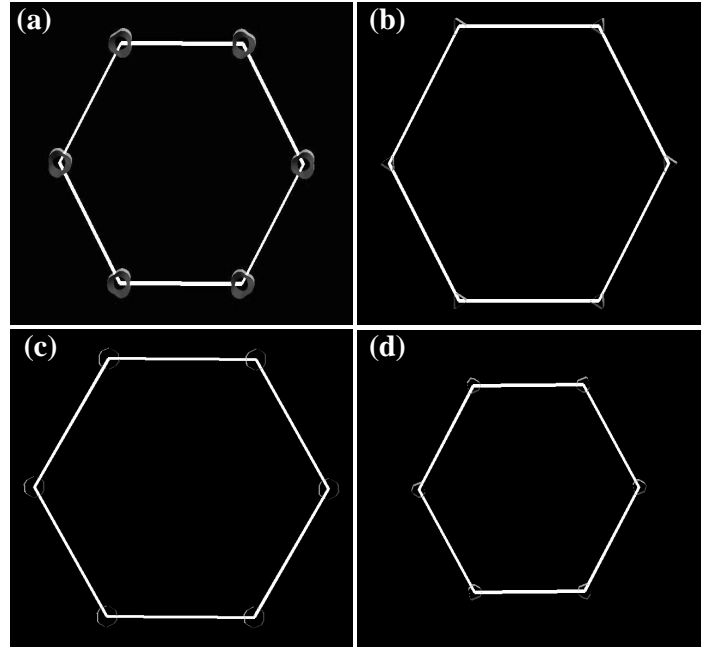

FIG. 3: Top view of the Fermi surfaces of (a) graphite, (b) monolayer graphene, (c) bilayer graphene, and (d) multilayer graphene.

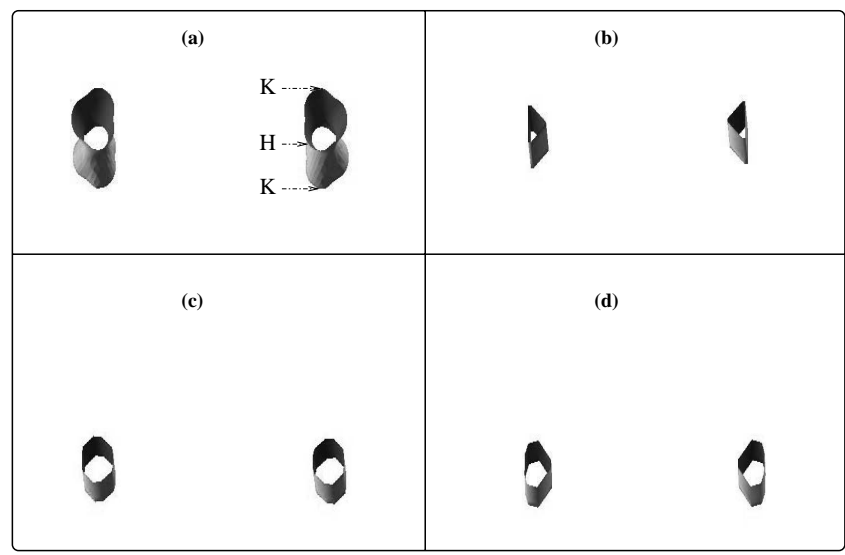

FIG. 4: Diagrams showing details of the full extent of the Fermi surfaces, of (a) graphite, (b) monolayer graphene, (b) bilayer graphene, and (d) multilayer graphene.

\section{III.2. Monolayer graphene}

The electronic band structure of graphene is shown in Fig. 1(b). Due to only two basis atoms in graphene there is only one pair of $\pi / \pi^{*}$ bands, which is degenerate at the $\mathrm{K}$ point, coinciding with the Fermi level. This means that this band is partially filled at the $\mathrm{K}$ point, and thus is contributed by both electron-like and hole-like states. Figure 2(b) shows that the dispersion of this band on the planar Brillouin zone around the K point (i.e. close to the Fermi level) is entirely linear (Dirac like) for both electrons and holes. The presently computed dispersion curve for the $\pi$ band agrees very well with the angle-resolved photoemission measurements made by Ohta et al [24]. The small level of disagreement between theory and experiment with regards to the energy locations at various in-plane wave vectors is well within the error margin of the experimental measurements. Our results are also in very good agreement with the results obtained by Latit and Henrard [25] who used an almost identical theoretical and computational method.

From our calculations of the in-plane dispersion curves slightly above and slightly below the Fermi energy we have estimated velocity results of $1.11 \times 10^{6} \mathrm{~m} / \mathrm{s}$ and $1.04 \times 10^{6}$ $\mathrm{m} / \mathrm{s}$ for electrons and holes, respectively. We attribute the difference between the hole and electron velocities of approximately $6 \%$ to the asymmetry of their band states. These results are in excellent agreement with the cyclotron resonance study by Deacon et al. [3] who measured the electron velocity of $1.093 \times 10^{6} \mathrm{~m} / \mathrm{s}$ and estimated the hole velocity to be $5 \%$ lower than the electron velocity. Our calculated electron velocity is also in excellent agreement with the value of $1.1 \times 10^{6} \mathrm{~m} / \mathrm{s}$ deduced by Jiang et al. [26] using infrared spectroscopic studies.

The Fermi surface for the monolayer graphene is shown in Fig. 3(b). Although the Fermi surface for an ideal graphene sheet has an area of zero size, the finite size of the plotted shape is due to the thermal broadening in the calculations. The resulting shape of the Fermi surface is a triangle centred at the $\mathrm{K}$ points in the Brillouin zone. As our calculations have been made using a supercell geometry, the resulting Fermi surface extends normal to the graphene sheet with both ends being triangles of equal dimensions. A clearer picture of the computed shape is shown in Fig. 4(b).

\section{III.3. Bilayer graphene}

We have modelled the bilayer graphene using the symmetric double layer (i.e. the Bernal, or $\mathrm{AB}$, stacking of graphite) arrangement. Due to a basis of four atoms, there are two pairs of $\pi / \pi^{*}$ bands. Consistent with previous theoretical works $[25,27]$, we find that similar to the monolayer graphene, the bilayer graphene is also a zero-gap semiconductor with the inner pair of the $\pi / \pi^{*}$ bands being degenerate at the $\mathrm{K}$ point. There is an energy gap of $0.8 \mathrm{eV}$ at the $\mathrm{K}$ point between the outer pair of the $\pi / \pi^{*}$ bands. This splitting arises from the interaction between the two layers. Band structure calculations for the bilayer graphene suggest that the in-plane dispersion relation of the inner $\pi^{*}$ electronic band at the $\mathrm{K}$ point has a mixture of linear and quadratic behaviour. The quadratic be- 
haviour of the dispersion relation is well accepted [2]. From our results we extract the carrier effective mass $0.022 m_{e}$ and velocity $1.10 \times 10^{6} \mathrm{~m} / \mathrm{s}$. The results from the tunnelling measurements done by Li et al. [28] suggest that the velocity of the carriers in bilayer graphene is $1.07 \times 10^{6} \mathrm{~m} / \mathrm{s}$, which is in fairly good agreement with our results.

The inter-layer interaction in the bilayer graphene results in a noticeable change in the shape of the Fermi surface around $\mathrm{K}$, the Brillouin zone edge point. The triangular Fermi surface for the monolayer graphene changes to a hexagonal shape. A detailed side view in Fig. 4(c) suggests that an identical hexagonal shape is maintained for both electrons and holes. This is rather different from the results of earlier tight-binding calculations (see, e.g. [2]) which have explained the change in the Fermi surface from the monolayer to the bilayer structure in terms of trigonal distortion (or warping).

\section{III.4. Multilayer graphene}

From the electronic band structure calculations for a seven-layer graphene system, we find that there are seven pairs of $\pi / \pi^{*}$ bands. The innermost $\pi / \pi^{*}$ pair is degenerate at the $\mathrm{K}$ point, making the system a zero-gap semiconductor. The outer six pairs of the $\pi / \pi^{*}$ bands are split, with the minimum and maximum gaps at $\mathrm{K}$ between the $\pi$ and $\pi^{*}$ bands being $0.14 \mathrm{eV}$ and $1.45 \mathrm{eV}$, respectively. The energy gap of $1.45 \mathrm{eV}$ between the outermost pair is close to that for bulk graphite. The splittings of the $\pi$ and $\pi^{*}$ bands are due to different amounts of interactions between the various atomic layers.

We further made calculations for the in-plane electronic band structure, close to the K edge of the Brillouin zone. We observe that the in-plane dispersion of the electronic band structure (shown in Fig. 2(d)) is quite similar to the dispersion in bulk graphite, but with a slightly more curvature close to the $\mathrm{K}$ edge. The values of the electron effective mass and velocity for the multilayer graphene are different compared with bulk graphite. The effective mass of the multilayer graphene is reduced by roughly $28 \%$, and the velocity, of $1.0 \times 10^{6} \mathrm{~m} / \mathrm{s}$, has increased by $6.4 \%$. Our calculated electron velocity agrees well with value deduced by Sadowski et al. [29] from their observed cyclotron resonance-like and electron-positron-like transitions in infrared transmission experiments. As is the case with the bilayer graphene, we could not find any experimental or other theoretical measurements of the electron effective mass for a multilayer graphene to compare or contrast our results with. The Fermi topology for the multilayer graphene shows a warped triangular shape, as shown in panel (d) of Figs. 3 and 4.

\section{III.5. Gradual changes from graphene to graphite}

From our work we are able to draw conclusions regarding the gradual development in the electronic properties with the dimensionality change from the two-dimensional graphene to the three-dimensional graphite. Within the energy range $\pm 2.0 \mathrm{eV}$ around the Fermi level, the occupied and unoccu- pied bands are of the orbital characters $\pi$ and $\pi^{*}$, respectively. The number of the pair of $\pi / \pi^{*}$ bands is directly related to the number of basis atoms in the unit cell: e.g. one for graphene, two for bilayer graphene and for bulk graphite, and seven for the seven-layer graphene. For a multilayer graphene there is noticeable splitting in the $\pi$ and $\pi^{*}$ bands, except for the innermost pair at the $\mathrm{K}$ point.

The in-plane electronic band dispersion is Dirac-like for graphene, a mixture of linear and quadratic for bilayer graphene and multilayer graphene, and quadratic for bulk graphite. The electron velocity decreases from the singlelayer graphene to the bilayer and multilayer graphene, and further to bulk graphite. The electron effective mass gradually increases from the bilayer graphene to multilayer graphene and then to bulk graphite. The shape of the Fermi surface changes as follows: triangle for graphene, hexagonal for bilayer graphene, warped triangle for multilayer graphene, and more warped triangle for bulk graphite. While the cross-section of the Fermi surface does not change along the KH direction for ideal monolayer graphene, bilayer graphene and multilayer graphene, it has a neck-like feature at the $\mathrm{KH} / 3$ point for bulk graphite.

\begin{tabular}{ccc}
\hline \hline Structure & Effective mass $\left(\mathrm{m}^{*} / \mathrm{m}_{e}\right)$ & Velocity $\left(10^{6} \mathrm{~m} / \mathrm{s}\right)$ \\
\hline Graphite & $0.043(0.045)^{[8,17]}$ & $0.94(0.91)^{[19]}$ \\
Monolayer graphene 'electrons' & 0.0 & $1.11(1.093-1.10)^{[3,26]}$ \\
Monolayer graphene 'holes' & 0.0 & 1.04 \\
Bilayer graphene & 0.022 & $1.10(1.07)^{[28]}$ \\
Multilayer graphene & 0.031 & $1.00(1.03)^{[29]}$ \\
\hline \hline
\end{tabular}

TABLE 1: Electron and hole effective masses and velocities in monolayer graphene, bilayer graphene, multilayer graphene, and graphite. Available experimentally measured results are presented in parentheses.

\section{SUMMARY AND CONCLUSION}

We have studied the electronic properties of graphene, bilayer graphene, multilayer graphene, and bulk graphite using the plane-wave pseudopotential method and the density functional theory within the local density approximation. The shape of the Fermi surface is found to be triangle for graphene, hexagonal for bilayer graphene, warped triangle for the multilayer graphene, and more warped triangle for bulk graphite. For graphite the cross-section of the Fermi surface has a neck-like feature at the $\mathrm{KH} / 3$ point. The in-plane electronic band structure near the zone edge $\mathrm{K}$ is Dirac-like for graphene, a mixture of linear and quadratic for bilayer graphene and multilayer graphene, and quadratic for bulk graphite. Our work suggests that the electron mass (velocity) gradually increases (decreases) with the thickness of the graphene layer. The electron effective mass is calculated to be $0.022 m_{e}, 0.031 m_{e}$, and $0.043 m_{e}$ for bilayer graphene, multilayer graphene, and graphite, respectively. The electron and hole velocities in monolayer graphene are $1.11 \times 10^{6}$ and $1.04 \times 10^{6} \mathrm{~ms}^{-1}$, respectively. The electron velocity gradually reduces to $1.10 \times 10^{6}, 1.0 \times 10^{6}$, and $0.94 \times 10^{6} \mathrm{~ms}^{-1}$ for bilayer graphene, multilayer graphene, and graphite, respectively. Our calculated values of the carrier velocities for these 
systems are in agreement with experimentally measured results. calculations reported here were performed using the University of Exeter's SGI Altix ICE 8200 supercomputer.

\section{Acknowledgements}

A. AlZahrani gratefully acknowledges financial support from King Abdulaziz University (KAU), Saudi Arabia. The

[1] R. J. Nicholas, A. Mainwood, and L. Eaves, Phil. Trans. R. Soc. A 366, 189 (2008).

[2] A. H. Castro Neto, F. Guinea, N. M. R. Peres, K. S. Novoselov and A. K. Geim, Rev. Mod. Phys. 81, 109 (2009).

[3] R. S. Deacon, K.-C. Chuang, R. J. Nicholas, K. S. Novoselov, and A. K. Geim, Phys. Rev. B 76, 081406 (2007).

[4] P. R. Wallace, Phys. Rev. 71, 622 (1947).

[5] J. W. McClure, Phys. Rev. 108, 612 (1957).

[6] S. J. Williamson, S. Foner, and M. S. Dresselhaus, Phys. Rev. 140, A1429 (1965).

[7] A. Zunger, Phys. Rev. B 17, 626 (1978).

[8] R. C. Tatar and S. Rabii, Phys. Rev. B 25, 4126 (1982).

[9] P. Hohenberg and W. Kohn, Phys. Rev. 136, B864 (1964).

[10] J. P. Perdew and A. Zunger, Phys. Rev. B 23, (1981) 5048.

[11] D. Vanderbilt, Phys. Rev. B 41, 7892 (1990).

[12] W. Kohn and L. Sham, Phys. Rev. 140, A1133 (1965).

[13] H.J. Monkhorst and J.D. Pack, Phys. Rev. B 13, 5189 (1976).

[14] M. Methfessel and A. T. Paxton, Phys. Rev. B 40, 3616 (1989).

[15] M. T. Yin and M. L. Cohen, Phys. Rev. B 29, 6996 (1984).

[16] M. C. Schabel and J. L. Martins, Phys. Rev. B 46, 7185 (1992).

[17] F. Rozploch, J. Patyk, and J. Stankowski, Acta. Phys. Pol. 112, 557 (2007).
[18] D. E. Soule, IBM J. Res. Dev. 8, 268 (1964).

[19] S. Y. Zhou, G.-H. Gweon, J. Graf, A. V. Fedorov, C. D. Spataru, R. D. Diehl, Y. Kopelevich, D.-H. Lee, S. G. Louie, and A. Lanzara, Nat. Phys. 2, 595 (2006).

[20] I. A. Luk'yanchuk and Y. Kopelevich, Phys. Rev. Lett. 93, 166402 (2004).

[21] I. A. Luk'yanchuk and Y. Kopelevich, Phys. Rev. Lett. 97, 256801 (2006).

[22] J. C. Slonczewski and P. R. Weiss, Phys. Rev. 109, 272 (1958).

[23] J. A. Woollam, Phys. Rev. B 3, 1148 (1970).

[24] T. Ohta, A. Bostwick, J. L. McChesney, T. Seyller, K. Horn, and E. Rotenberg, Phys. Rev. Lett. 98, 206802 (2007).

[25] S. Latil and L. Henrard, Phys. Rev. Lett. 97, 036803 (2006).

[26] Z. Jiang, E. A. Henriksen, L. C. Tung, Y.-J. Wang, M. E. Schwartz, M. Y. Han, P. Kim, and H. L. Stormer, Phys. Rev. Lett. 98, 197403 (2007).

[27] H. Min, B. Sahu, S. K. Banerjee, and A. H. MacDonald, Phys. Rev. B 75, 155115 (2007).

[28] G. Li and E. Y. Andrei, Nat. Phys.3, 623 (2007).

[29] M. L. Sadowski, G. Martinez, and M. Potemski, Phys. Rev. Lett. 97, 266405 (2006). 